Editorial Office Notes:

RES-16-148.R1

ORIGINAL ARTICLE

Received 22 February 2016

Invited to revise 13 April 2016

Revised 23 May 2016

Accepted 9 June 2016

Associate Editor: Conroy Wong 


\section{Bronchiectasis in yellow nail syndrome}

Dr Woodfield $\mathrm{G}^{1}$, Dr Nisbet $\mathrm{M}^{1}$, Dr Jacob J ${ }^{2}$, Dr Mok W${ }^{2}$, Dr Loebinger MR ${ }^{1}$, Prof Hansell $\mathrm{DM}^{2}$, Prof Wells $\mathrm{AU}^{3}$, Prof Wilson $\mathrm{R}^{1}$

1. Host Defence Unit, Royal Brompton Hospital, London,

2. Radiology Department, Royal Brompton Hospital, London,

3. Interstitial Lung Disease, Royal Brompton Hospital, London, UK

\section{Correspondence:}

Professor R Wilson MD, FRCP

Consultant Physician

Host Defence Unit

Royal Brompton Hospital

Sydney Street

London SW3 6NP

UK

Email: $\quad$ r.wilson@rbht.nhs.uk

\section{Summary at a glance}

Bronchiectasis in yellow nail syndrome (YNS) has distinct features which influence clinical presentation and management. Bronchiectasis in YNS is milder than in idiopathic bronchiectasis, with less upper and middle lobe disease, but increased mucus plugging. A better understanding of aetiology may influence future research and management decisions. 


\begin{abstract}
Background and objective: Yellow nail syndrome (YNS) is a rare and poorly described disease process. In this case control study, clinical features and findings on high resolution computed tomography $[\mathrm{HRCT}]$ were compared with idiopathic bronchiectasis (IBx).
\end{abstract}

Methods: A review of all patients attending an adult bronchiectasis clinic between 2007 and 2013 identified 25 YNS patients. IBx patients were matched in a 2:1 ratio for age, duration of symptoms and gender.

Results: Median age of onset was 53 years. There were 12 male and 23 Caucasian YNS patients. Respiratory manifestations included chronic productive cough (100\%), chronic rhinosinusitis (88\%), pleural effusions (20\%), lymphoedema (12\%). Chest symptoms preceded yellow nails in the majority (68\%). Abnormal nails persisted at follow up in 23/25 patients but improved in 14 .

In both disorders, there was symmetrical, predominantly lower lobe bronchiectasis on HRCT. Extent $(\mathrm{p}=0.04)$, severity $(\mathrm{p}=0.03)$ and bronchial wall thickness $(\mathrm{p}=0.05)$ scores were lower in YNS, with less upper and middle lobe disease. Multivariate analysis showed an independent association with increased mucus plugging in YNS. There was a similar prevalence of Pseudomonas aeruginosa infection and mild lung function abnormalities.

Conclusion: Bronchiectasis in YNS is less severe than IBx but is associated with increased mucus plugging, onset is in middle age and there is no female predominance. Treatment targeted at improved secretion clearance may improve both chest and nail symptoms, with consideration of longterm macrolide antibiotics. 
Keywords: yellow nail syndrome, Pseudomonas aeruginosa, lymphoedema, pleural effusion, idiopathic bronchiectasis

Short title: Bronchiectasis in yellow nail syndrome 


\section{INTRODUCTION}

Yellow Nail Syndrome (YNS) was initially described by Samman and White in 1964 and the literature currently documents approximately 150 cases $^{1}$. It is a rare clinical syndrome characterised by a triad of yellow dystrophic nails, lymphoedema, and pulmonary manifestations. These include chronic cough with sputum, recurrent respiratory infections, pleural effusions, bronchiectasis and rhinosinusitis. It has been previously accepted that two of the triad are adequate for diagnosis ${ }^{2}$. The complete triad occurs in one third of patients ${ }^{3}$ where symptoms may occur years apart ${ }^{4}$. The characteristic nail changes in YNS include yellow-green discolouration, slow growth, cross-ridging, loss of cuticle, over-curvature, onycholysis and shedding ${ }^{1}$.

The aetiology of YNS remains undefined however the pathogenesis may involve impaired lymphatic drainage ${ }^{5}$. Lymphangiography has shown hypoplastic lymphatic systems in the legs and lungs of some patients ${ }^{1,5}$. This finding has not been confirmed by lymphoscintigraphic studies and, given the reversibility in some, the underlying cause may be functional rather than anatomical ${ }^{5}$. A small subset has hypoalbuminaemia caused by enteric albumin loss suggesting increased microvascular permeability ${ }^{6}$. The cause of bronchiectasis is unclear but may relate to dysfunctional lymphatics and impaired secretion drainage, with subsequent increased infection risk.

In 2008 Maldonado et al described 41 patients with YNS, mean age of onset 61 years. 18 patients $(44 \%)$ had mainly symmetrical lower lobe bronchiectasis ${ }^{7} .17(41 \%)$ had chronic rhinosinusitis. By comparison a study of 165 consecutive bronchiectasis patients referred to a single centre contained 4 patients with YNS and 43 with IBx. The 
mean age of onset of IBx was 43 years with female predominance (65\%), with a predominantly lower lobe bronchiectasis and chronic rhinosinusitis ${ }^{8}$.

Patients with different bronchiectasis aetiologies have characteristic CT scan appearances and clinical features. This case control study of patients referred to a tertiary speciality hospital aims to further define the respiratory features of YNS by comparing radiological findings, lung function and microbiology with a matched cohort of IBx patients.

\section{METHODS}

YNS patients were identified by electronically searching all Royal Brompton Hospital adult bronchiectasis clinic letters from 2007 to 2013. Patients were initially referred for chronic productive cough with suspected or previously confirmed bronchiectasis, with or without yellow nails. Diagnosis of YNS was made (often subsequently) after development of yellow nails. Clinical features, microbiology, radiology, lung function, progress and follow up duration were recorded. All patients had been investigated using a protocol previously published [8]. This includes blood investigations to exclude known aetiologies; nasal nitric oxide as a test for primary ciliary dyskinesia (followed by light and electron microscopy of nasal epithelium if indicated); sweat test (and/or genotyping) for cystic fibrosis ${ }^{8}$. The study was an anonymised retrospective review of cases and neither patient consent nor ethical approval was sought. All patients attending the Royal Brompton Hospital are informed about the use of patient data for continuing research at the hospital. 
IBx patients were defined as having had no history of serious infections predating bronchiectasis, with normal or negative tests for other bronchiectasis aetiologies ${ }^{8}$. The IBx comparison group was formulated by matching IBx patients to YNS patients in a 2:1 ratio, matched for age (within 10 years), duration of symptoms (within 5 years) and, for lung function comparison, gender.

High resolution computed tomography (CT) scans were performed on initial assessment. $1.5 \mathrm{~mm}$ sections were obtained at $10 \mathrm{~mm}$ intervals, supine and at full inspiration. Bronchiectasis was defined according to accepted CT criteria ${ }^{9}$. All CT scans were independently reviewed by the same two experienced observers, blinded to clinical information. Observers awarded scores (grades) per lobe (lingula regarded as a separate lobe) for eight variables: extent of bronchiectasis, severity of bronchial dilation, bronchial wall thickness, mucus plugging, tree-in-bud pattern, mosaicism, consolidation and septal thickening. Full scoring criteria are described in Supplementary Appendix S1, and are similar to previous studies ${ }^{10-11}$. After initial scoring, a consensus score was agreed between observers in cases with $>1$ point discrepancy, where one result was zero, or where there was a $>15 \%$ discrepancy for Mosaicism or Consolidation. Consensus scores were used for data analysis.

\section{Statistical analysis}

HRCT variables were evaluated as follows:

1. Global scores (per scan) for all variables were computed as the sum of lobar grades, with the exception of mosaic attenuation. See Appendix S1 for details of scoring system. For example, the maximum grade per lobe for "extent" was 3, therefore the maximum global extent score was 18 (assuming 6 lobes). 
Global mosaic attenuation scores were calculated as the mean of lobar scores, as mosaic attenuation was a continuous variable rather than a grade (quantified as the percentage of total lung volume in each lobe). Mean scores for the two observers were evaluated in all analyses of global variables. Lobar grades were recorded in a table as shown in Appendix S2, for each observer.

2. Septal thickening and consolidation were seldom present and were not evaluated further.

3. Regional scores were computed as upper lobe, middle lobe and lower lobe scores using the same approach as for global scores (i.e. mean lobar scores for mosaic attenuation, summed scores for all other variables).

Group comparisons for all variables were made using the Wilcoxon rank sum test. A $\mathrm{p}$ value $<0.05$ indicated statistical significance. Stepwise logistic regression was used to identify independent HRCT morphologic differences between YNS and IBx.

Lung function was compared between diseases, where YNS and IBx patients were also sex matched (as well as for age and symptom duration). This meant that the matched IBx lung function group and radiology group were not necessarily formed of the same IBx patients. However, matched YNS/IBx patients were kept the same where possible; 28 of the idiopathic patients used for lung function comparison were also used for CT comparison (this meant that there were 16 IBx patients that featured only in the PFT comparison group, and 10 that featured only in the CT comparison group). The YNS group was consistent throughout. The lung function matched IBx group was the comparator group used to compare microbiology findings between diseases (as opposed the IBx radiology matched group used for CT comparison). Lung function readings and 
microbiology data were recorded at initial assessment and at every subsequent visit (in order to record any subsequent $P$. aeruginosa infection). Lung function data were compared using an unpaired student t-test. Categorical variables, namely presence or absence of $P$. aeruginosa, were compared using a chi-squared test. $\mathrm{P}<0.05$ was regarded as being statistically significant.

\section{RESULTS}

Twenty five patients with YNS attended clinics at the Royal Brompton Hospital between 2007 and 2013. The median referral age of YNS was 58.2 years (range 11.877.7), and median age of first symptoms was 53 (range 4-75). 12 were male (48\%). 23/25 (92\%) were Caucasian, from the UK and Ireland. One patient was from Iraq with Arabic origins, the other was Asian from Sri Lanka. All presented to the Royal Brompton with chronic cough and recurrent infections. During follow up, 21 patients suffered from chronic sinusitis, 10 had wheeze. Three patients had lymphoedema of lower limbs, one with left arm involvement. Five patients had a history of pleural effusion. Supplementary Tables S1 and S2 describe YNS and IBx individual patient characteristics in more detail.

$22 / 25$ patients $(88 \%)$ had abnormal nails at referral, with the remaining 3 patients developing yellow nails within two years. In the majority of patients $(17,68 \%)$, chest symptoms preceded nail changes. Yellow nails had preceded chest symptoms by one year in two patients, and occurred simultaneously in six. Two patients had a history of 
malignancy (breast and melanoma), five patients had reflux, four had coronary artery disease and two had cardiac failure. One patient had asbestos plaques. The proportion of ex-smokers was similar between YNS and IBx (32\% and 29\% respectively). None were current smokers.

Immunoglobulins were normal in all. Two patients had lymphopenia (lymphocyte count of $0.7 \times 10^{9} / \mathrm{L}$ and $\left.1.0 \times 10^{9} / \mathrm{L}\right)$. Four patients had low albumin levels of $26-34 \mathrm{~g} / \mathrm{L}$. Seven patients had elevated IgE levels (>150 IU/mL). Two of these were diagnosed with aspergillus sensitivity, with Aspergillus fumigatus RAST results of 14.7 and 9.74 IU/mL. Neither of these patients had peripheral eosinophilia nor clinical/radiological evidence of ABPA.

The median follow-up was 6.2 years (range 0 to 19 years) in the YNS cohort, and 4.9 years (range 0 to 14.7 years) in the IBx cohort. Zero follow up was recorded where patients were seen once only (1/25 YNS, 5/44 IBx). Initial referral dates ranged between 1992-2013 for YNS, and 2001-2012 for IBx patients.

\section{Microbiology}

At initial referral, sputum cultured $P$. aeruginosa in 5/22 YNS patients (23\%), Haemophilus influenzae in five (23\%), Streptococcus pneumoniae in two (9\%), and Moraxella catarrhalis in one patient (5\%). Nine (41\%) patients had no significant bacterial growth. 3 patients' initial results were unavailable. No mycobacteria were cultured. By comparison, 12/44 (27\%) IBx patients cultured P. aeruginosa. During follow up, 9 further YNS and 12 further IBx patients became colonised (64\% and 55\% of cohort respectively). There was no significant difference in incidence of $P$. 
aeruginosa at presentation ( $\mathrm{p}=0.69)$, or during follow up $(\mathrm{p}=0.48)$ between diseases. Table 1 compares microbiology between diseases.

\section{Lung function studies}

Lung function studies showed YNS to be a mildly obstructive lung disease, with no significant difference in readings compared to IBx (Table 2).

Within the YNS group, patients colonised with $P$. aeruginosa at presentation had a statistically lower mean FEV1 $(\mathrm{p}=0.013)$ and FVC $(\mathrm{p}=0.042)$ than those without colonisation.

\section{Radiology}

Nineteen YNS patients had CT scans available, where seventeen showed bronchiectasis. The remaining two patients scored zero for extent and severity of bronchiectasis. However, both had bronchial wall thickening, chronic daily sputum production and recurrent infections, with bronchiectasis diagnosed clinically. One patient's CT scan had been reported as mild bilateral lower lobe bronchiectasis by the radiologist reporting it originally; the second patient had right lower lobe bronchiectasis on a repeat scan 2 years later.

YNS patients had significantly lower extent $(\mathrm{p}=0.04)$, severity $(\mathrm{p}=0.03)$ and bronchial wall thickness $(\mathrm{p}=0.05)$ scores than matched IBx patients, see Table 3. Differences in scores for tree in bud, mosaicism and mucus plugging did not reach statistical significance on initial analysis (Wilcoxons signed rank test). Consolidation was present in only $4 / 19(21 \%)$ of YNS and 13/38 (34\%) of IBx patients. Septal thickening was present in only 6/19 (32\%) of YNS and 13/38 (34\%) of IBx patients. Median global 
scores for consolidation and septal thickening were zero, so they were not included in Table 3.

Examination of a stepwise logistic regression model (with the HRCT variables listed in Table 3 examined as initial covariates prior to stepwise evaluation) showed that when compared to IBx, YNS was independently associated with less bronchial wall thickness (OR 0.64; 95\% CI 0.46-0.87; $\mathrm{p}<0.005)$ and higher mucus plugging scores (OR 1.89; 95\% CI $1.10-3.25 ; \mathrm{p}=0.02)$ than IBx. Analyses of the distribution of abnormalities on HRCT revealed that when compared to IBx, YNS was characterised by lower scores for the extent and severity of bronchiectasis and overall bronchial wall thickness (all $\mathrm{p}<0.05)$. However, lower lobe scores taken in isolation did not differ between the two diseases for any HRCT variable. Thus, compared to IBx, YNS is associated with sparing of the upper and middle lobes, with the abnormalities being present in the lower lobes.

\section{Management}

All patients received physiotherapy tuition at presentation and throughout follow up. Low dose antibiotic prophylaxis was introduced in $20(80 \%)$ of YNS patients because of poor symptom control and/or four exacerbations or more per year. Three of these stopped antibiotics due to intolerance, side effects or non-response, leaving 17 on longterm prophylaxis. Prophylaxis was oral azithromycin in 14/17 (82\%) (usually 250mg three times weekly). This improved chest symptoms in the majority $(9 / 14=64 \%)$ of this group. One patient colonised with $P$. aeruginosa was taking colomycin nebulisers additionally as dual prophylaxis. $3 / 17$ took other forms of antibiotic prophylaxis; 2 took colomycin alone (intolerant to azithromycin) with improvement in symptoms, and one 
on regular erythromycin had no improvement. For the YNS group as a whole, 16 (64\%) had a subjective improvement in chest symptoms since first review. Eleven of these were taking regular antibiotic prophylaxis and five patients improved with physiotherapy alone. Figures 1a and 1b shows a CT scan of a YNS patient before and after treatment with a long course of clarithromycin $250 \mathrm{mg}$ twice daily and inhaled colomycin 1million units twice daily, started when pseudomonas was isolated.

23/25 patients had persisting nail changes at the time of last review, where 17 had improved over time. Seven of these also had improved chest symptoms over time. Resolution of nail changes occurred in two patients with improvement in chest symptoms (complete resolution in one). Figures $2 \mathrm{a}$ and $2 \mathrm{~b}$ shows an example of one patient's nail changes before and after treatment with physiotherapy and episodic antibiotics during infective exacerbations. 


\section{DISCUSSION}

This study investigates YNS patients referred to a tertiary hospital respiratory clinic, and compares them to matched IBx patients. IBx was chosen as the comparator group because both groups have normal or negative tests for other aetiologies, present in a similar way and are currently only differentiated by nail changes. A better understanding of aetiology may influence future research and management decisions. A recent multicentre study of 1258 bronchiectasis patients determined the aetiology in $60 \%$, where this knowledge changed the patient's management in $13 \%$ of cases $^{12}$. Additionally, the radiological and clinical findings in YNS bronchiectasis have been poorly defined. In Varney et al's series of 17 YNS patients, all had chronic cough and the majority had rhinosinusitis $(83 \%)^{13}$. Lymphoedema was present in $76 \%$, compared to only $36 \%$ with lymphatic dysfunction in our series. Hoque et al. described eleven YNS patients of whom only six had bronchiectasis ${ }^{14}$. Comparable with our cohort the mean age of onset of symptoms was 53 years (range 27 to 69) but in contrast they reported lymphoedema in $46 \%$ of cases and only $27 \%$ had sinusitis. A recent study of 165 consecutive bronchiectasis patients ( 43 with IBx) found the age average of onset of IBx was $43,65 \%$ women ${ }^{8}$. Other studies have shown similarly younger age of onset for IBx and female predominance ${ }^{15-16}$. In our YNS cohort the average age of onset was 53 years, with no gender predominance.

YNS has been associated with other conditions including autoimmune disorders [4], immunodeficiency states ${ }^{17-18}$, nephrotic syndrome ${ }^{19}$, Guillain-Barre syndrome ${ }^{20}$, drugs (D-penicillamine, gold) and cancers $4,6,17,19,21-28$. Three of our patients had a history of malignancy. YNS has also been reportedly associated with lymphopenia, low IgA levels and hypogammaglobulinaemia ${ }^{29}$. Two patients in our series had lymphopenia but 
immunoglobulin levels were normal in all. These previously described associations of YNS were therefore not seen in our cohort of patients presenting to a chest clinic.

The notable CT finding of YNS in our study was symmetrical predominantly lower lobe bronchiectasis, with significantly less upper and middle lobe disease, and increased mucus plugging compared to IBx. Our cohort of YNS patients had significantly milder disease than IBx in terms of total extent, severity and bronchial wall thickening. However, this may be explained by disease being restricted to the lower lobes in YNS. We therefore hypothesise that bronchiectasis in YNS is a disorder of delayed mucociliary clearance due to abnormal lymphatic structure/function, where gravity influences lower lobe disease development. However, other factors such as frequency and severity of infections and the presence of other pro-inflammatory influences (such as aspergillus sensitivity in two patients), will also determine bronchiectasis development. Previous CT findings in YNS include one case series of four patients all with bronchiectasis primarily in the middle lobe, lingula and lower lobes ${ }^{30}$. Maldonado et al reported 41 YNS patients where 18 had bilateral lower lobe bronchiectasis on $\mathrm{CT}^{7}$. A possible explanation for milder disease in YNS could be earlier referral (and therefore treatment) due to yellow nail development. This explanation is however unlikely because YNS and IBx patients were matched by length of symptoms at the time of CT/lung function tests.

The YNS group as a whole had mild abnormalities of lung function, although patients with $P$. aeruginosa had significantly poorer FEV1 readings at presentation $(\mathrm{p}=0.013)$. The association with $P$. aeruginosa with more severe airflow limitation has been noted in bronchiectasis previously ${ }^{31-32}$. Lung function was comparable to the IBx cohort, as 
was incidence of $P$. aeruginosa both at presentation and subsequently. This was surprising considering less severe and less widespread bronchiectasis in YNS. One explanation could be increased mucus plugging predisposing to bacterial colonisation, or possibly higher use of antibiotic prophylaxis in this group.

Longterm prophylaxis with azithromycin improved chest symptoms (cough, sputum and exacerbation frequency) in the majority $(64 \%)$ of the YNS cohort taking it. This suggests that it is a good choice where symptom control is poor with physiotherapy and rescue antibiotics. Improvement with clarithromycin has been described in two case studies of $\mathrm{YNS}^{33-34}$. However, one study focussed on nail improvement, whilst the second described a case of eosinophilic bronchial disease in YNS, unlike in our cohort.

There are some limitations to our study. YNS and IBx cohorts were matched for age and length of symptoms, therefore patient recollection was used to determine symptom onset date. The use of a small (five year) margin attempts to minimise poor recollection or individual variability in perception of symptoms. There may be some patient selection bias, as YNS patients may be referred earlier due to yellow nails rather than for problematic respiratory symptoms. However, the majority of YNS patients in our cohort were undiagnosed at referral. This study did not assess disease improvement objectively, and relied on clinic letter reports of symptoms. This is a small cohort of patients, as YNS is rare and we are a single centre. However, being a tertiary respiratory centre has enabled us to match IBx patients

In conclusion, this study shows YNS as a distinct aetiology of bronchiectasis that may relate to impaired mucus clearance. The bronchiectasis in YNS is usually mild and 
involves predominantly lower lobes, often presenting at an older age than IBx. Management should target mucus clearance. Longterm macrolide antibiotics may provide symptomatic relief, and in some cases led to resolution of dystrophic nails. Patients should be screened for impaired immunity, aspergillus sensitivity and malignancy, although these findings were uncommon in our cohort. 
Table 1- Comparison of microbiology between diseases at first clinic appointment

\begin{tabular}{|l|l|l|}
\hline $\begin{array}{l}\text { Initial Sputum culture } \\
\text { results }\end{array}$ & $\begin{array}{l}\text { Yellow Nail Syndrome } \\
\text { Frequency of culture } \\
\text { result }\end{array}$ & $\begin{array}{l}\text { Idiopathic Bx frequency } \\
\text { Frequency of culture } \\
\text { result }\end{array}$ \\
\hline $\begin{array}{l}\text { No growth/ Upper } \\
\text { respiratory tract flora }\end{array}$ & 9 & 8 \\
\hline $\begin{array}{l}\text { Pseudomonas } \\
\text { aeruginosa }\end{array}$ & 5 & 12 \\
\hline $\begin{array}{l}\text { Haemophilus } \\
\text { Influenzae }\end{array}$ & 5 & 12 \\
\hline $\begin{array}{l}\text { Streptococcus } \\
\text { pneumonia }\end{array}$ & 2 & 1 \\
\hline Moraxella cattarhalis & 1 & 4 \\
\hline $\begin{array}{l}\text { Stenotrophomonas } \\
\text { maltophilia }\end{array}$ & 0 & 1 \\
\hline Staphylococcus aureus & 0 & 3 \\
\hline Coliforms & 0 & 1 \\
\hline Serratia marcesens & 0 & 1 \\
\hline $\begin{array}{l}\text { Beta haemolytic } \\
\text { streptococcus }\end{array}$ & 0 & 1 \\
\hline & $\begin{array}{l}\text { Total samples =22 (3 } \\
\text { patients not available) }\end{array}$ & Total samples = 44 \\
\hline
\end{tabular}

The IBx group used for comparison was the IBx lung function test group (patients varied very slightly to the IBx CT comparison group) 
Table 2- Lung function tests in Yellow Nail Syndrome and Idiopathic Bronchiectasis

\begin{tabular}{|l|c|c|c|}
\hline & Yellow Nail & Idiopathic & \\
\hline Mean FEV1\% of & Syndrome & $\mathrm{n}=44$ & P value \\
predicted value & $83.86(15.80)$ & $76.50(26.91)$ & 0.251 \\
(Standard deviation) & & & \\
\hline Mean FEV1/FVC ratio & & & 0.413 \\
(Standard deviation) & $0.70(0.10)$ & $0.67(0.17)$ & 0.740 \\
\hline Mean TLCO (transfer & & & \\
factor) \% of predicted & $76.43(15.52)$ & $77.90(16.70)$ & \\
(Standard deviation) & & & \\
\hline
\end{tabular}

Student $t$ test was used to compare data sets. 
Table 3 High Resolution Computed Tomogram variables compared between Yellow Nail Syndrome and Idiopathic Bronchiectasis.

\begin{tabular}{|c|c|c|c|}
\hline $\begin{array}{l}\text { HRCT variables } \\
\text { (max score per } \\
\text { scan) }\end{array}$ & $\begin{array}{l}\text { YNS -19 } \\
\text { patients } \\
\text { Median CT } \\
\text { score } \\
\text { (range) }\end{array}$ & $\begin{array}{c}\text { IBx -38 patients } \\
\text { Median CT } \\
\text { score } \\
\text { (range) }\end{array}$ & $P$ value \\
\hline $\begin{array}{c}\text { Extent of } \\
\text { bronchiectasis (18) }\end{array}$ & $\begin{array}{c}4 \\
(0-14)\end{array}$ & $\begin{array}{c}8 \\
(0-16)\end{array}$ & 0.04 \\
\hline $\begin{array}{c}\text { Severity of } \\
\text { bronchiectasis (24) }\end{array}$ & $\begin{array}{c}5.5 \\
(0-13.5)\end{array}$ & $\begin{array}{c}8 \\
(0-18.5)\end{array}$ & 0.03 \\
\hline Wall thickness (24) & $\begin{array}{c}5.5 \\
(1-13)\end{array}$ & $\begin{array}{c}6.5 \\
(1-17)\end{array}$ & 0.05 \\
\hline Tree in bud (12) & $\begin{array}{c}2.5 \\
(0-7)\end{array}$ & $\begin{array}{c}2.75 \\
(0-8.5)\end{array}$ & 0.73 \\
\hline $\begin{array}{c}\text { Mucus } \\
\text { Plugging (12) }\end{array}$ & $\begin{array}{c}2.0 \\
(0-6)\end{array}$ & $\begin{array}{c}2.0 \\
(0-8)\end{array}$ & 0.80 \\
\hline $\begin{array}{l}\text { Mosaic attenuation } \\
\qquad(100 \%)\end{array}$ & $\begin{array}{c}20 \\
(0-65 \%)\end{array}$ & $\begin{array}{c}25 \\
(0-90 \%)\end{array}$ & 0.86 \\
\hline
\end{tabular}

HRCT variables including maximum scores are expressed as the sum of lobar scores for each patient, with the exception of mosaic attenuation (quantified as percentage of lung volume). All lobar scores were averaged for two observers, before summing. See Supplementary Appendix S1 for details of how each lobe was scored. 


\section{Figure legends}

Figure 1- (A) 57 year old female with YNS at presentation. HRCT section through the lower lobes showing cylindrical bronchiectasis, particularly in the left lower lobe where there is an associated tree-in-bud pattern; there are also small foci of consolidation in the left lower lobe and lingual. (B)- In the same patient 18 months later, after treatment with a long course of clarithromycin $250 \mathrm{mg}$ twice daily and inhaled colomycin 1 million units twice daily, The appearances in the left lower lobe have improved with resolution of the tree-in-bud pattern and consolidation.

Figure 2 - (A) 54 year old man with yellow nails at first presentation, demonstrating discolouration, ridging and shedding. (B) - The same patient 1.5 years later demonstrating complete resolution of nail changes after treatment with physiotherapy alone. 


\section{REFERENCES}

1. Samman P, White W. The "Yellow nail syndrome". Br J Dermatol. 1964;76: 153-7.

2. Hershko A, Hirshberg B, Nahir M et al. Yellow Nail Syndrome. Postgrad Med J. 1997;73: 466-8.

3. Emerson P. Yellow nails, lymphoedema, and pleural effusions. Thorax. 1966; 21: 247-53.

4. Gupta A, Davies G, Haberman H. Yellow nail syndrome. Cutis. 1986; 37: 371-4.

5. Bull R, Fenton D, Mortimer P. Lymphatic function in the yellow nail syndrome. Br J Dermatol. 1996; 134: 307-12.

6. D’Alessandro A, Muzi G, Monaco A, Filiberto S, Barboni A, Arbritti G. Yellow nail syndrome: does protein leakage play a role? Eur Resp J. 2001; 17: 149-52.

7. Maldonado F, Tazelaar H, Wang C, Ryu J. Yellow nail syndrome: analysis of 41 consecutive patients. Chest. 2008: 134: 375-381

8. Shoemark A, Ozerovitch L, Wilson R. Aetiology in adult patients with bronchiectasis. Respir Med. 2007; 101: 1163-70.

9. Naidich DP, McCauley DI, Khouri NF, Stitik FP, Siegelman SS. Computed tomography of bronchiectasis. J Comput Assist Tomogr. 1982; 6: 437-44.

10. Horsley AR, Davies JC, Gray RD et al. Changes in physiological, functional and structural markers of cystic fibrosis lung disease with treatment of a pulmonary exacerbation. Thorax. 2013; 68: 532-539

11. Reiff DB, Wells AU, Carr DH, Cole PJ, Hansell DM. CT findings in bronchiectasis: limited value in distinguishing between idiopathic and specific types. Am J Roentgenol. 1995;165: 261-7.

12. Lonni S, Chalmers J, Goeminne P, McDonnell M, Dimakou K, De Soyza A, Polverino E, Van De Kerkhove C, Rutherford R, Davison J, Rosales E, Pesci A, 
Restrepo M, Torres A, Stefano A. Etiology of non-cystic fibrosis bronchiectasis in adults and its correlation to disease severity. Ann Am Thorac Soc. 2015; 12: 1764 1770.

13. Varney V, Cumberworth V, Sudderick R, Durham S, Mackay I. Rhinitis, sinusitis and the yellow nail syndrome: a review of symptoms and response to treatment in 17 patients. Clin Otolaryngol. 1994; 19: 237-40.

14 . Hoque S, Mansour S, Mortimer P. Yellow nail syndrome: not a genetic disorder? Eleven new cases and a review of the literature. Brit J Dermat. 2007; 1230-4.

15. Smith C. Prevots D, Adjemian J , Seitz A, Daniels M, Czaja C , Milla C , Hall D , Holland S, Knowles M, Olivier K. Characterization Of Idiopathic Bronchiectasis In Patients With And Without Pulmonary Nontuberculous Mycobacterial Disease. Am J Respir Crit Care med. 2013;187; A4535

16. S. Fuschillo, A. De Felice and G. Balzano. Mucosal inflammation in idiopathic bronchiectasis: cellular and molecular mechanisms. Eur Respir J. 2008; 31: 396406

17. Siegelman S, Heckman B, Hasson J. Lymphedema, pleural effusions and yellow nails: associated immunologic deficiency. Dis Chest. 1969; 56: 114-7.

18. Scher R. Acquired immunodeficiency syndrome and yellow nails. J Am Acad Dermatol. 1988; 18: 758-9.

19. Yanez S, Val-Bernal J, Fernandez-Llaca H. Yellow nails and minimal change nephrotic syndrome. Nephron. 1999; 82: 180-2.

20. Woollons A, Darley C. Yellow nail syndrome following Guillian-Barre syndrome. Clin Exp Dermatol. 1997; 22: 253-4. 
21. Iqbal M, Rossoff L, Marzouf K, Steinberg H. Yellow nail syndrome: resolution of yellow nails after successful treatment of breast cancer. Chest. 2000; 117: 1516-8.

22. Guin J, Elleman J. Yellow Nail syndrome: possible association with malignancy. Arch Dermatol. 1979; 115: 734-5.

23. Thomas P, Sidhu B. Yellow nail syndrome and bronchial carcinoma. Chest. 1987; 92: 191.

24. Burrows N, Jones R. Yellow nail syndrome in association with carcinoma of the gall bladder. Clin Exp Dermatol. 1991; 16: 471-3.

25. Hiller E, Rosenow E, Olsen A. Pulmonary manifestations of the yellow nail syndrome. Chest. 1972; 61: 452-8.

26. Stosiek N, Paters K, Hiller D et al. Yellow nail syndrome in a patient with mycosis fungoides. J Am Acad Dermatol. 1993; 28: 792-4.

27. Seve P, Thieblemont C, Dumontet C, Boufia F, Arnaud P, Hequet O, Espinouse D, Salles G, Coiffier B. Skin lesions in malignancy. Case 3. Yellow nail syndrome in nonHodgkins lymphoma. J Clin Oncol. 2001; 19: 2100-1.

28. Ginarte M, Monteagudo B, Toribio J. Yellow nail syndrome and lung lymphoma. Clin Exp Dermatol. 2004; 29: 423-36.

29. Bokszczanin A, Levinson A. Coexistent yellow nail syndrome and selective antibody deficiency. Ann Allergy Asthma Immunol. 2003; 91: 496-500.

30. Wiggins J, Strickland B, Chung K. Detection of bronchiectasis by high-resolution computed tomography in the yellow nail syndrome. Clin Radiol. 1991; 43: 377-9.

31. Davies G, Wells A, Doffman S, Watanabe S, Wilson R. The effect of Pseudomonas aeruginosa on pulmonary function in patients with bronchiectasis. Eur Respir J. 2006; 28: 974-9. 
32. Evans SA, Turner SM, Bosch BL, Hardy CC, Woodhead MA. Lung Function in bronchiectasis: the influence of Pseudomonas aeruginosa. European Resp J. 1996; 9: $1601-4$

33. Suzuki M, Yoshizawa A, Sugiyama H, Ichimura Y, Morita A, Takasaki J, Naka G, Hirano S, Izumi S, Takeda Y, Hoji M, Kobayashi N, Kudo K. A case of yellow nail syndrome with dramatically improved nail discoloration by oral clarithromycin. Case Resp Dermatol. 2011; 3:251-8.

34. Toyoshima M, Chinda K, Suda T. A case of yellow nail syndrome associated with eosinophilic bronchial disease successfully treated with clarithromycin and budesonide. Nihon Kokyuki Gakkai Zasshi. 2005; 43: 508-12. 